\title{
3q26.2/EVI1 rearrangement is associated with poor prognosis in classical Philadelphia chromosome-negative myeloproliferative neoplasms
}

\author{
Zhihong $\mathrm{Hu}^{1}$, L Jeffrey Medeiros ${ }^{1}$, Wei Wang ${ }^{1}$, Zi Chen ${ }^{1}$, Guilin Tang ${ }^{1}$, Parsa Hodjat ${ }^{1}$, \\ $\mathrm{Su}$ Yang $^{1}$, Lianghua Fang ${ }^{1}$, Yan $\mathrm{Li}^{1}$, Srdan Verstovsek ${ }^{2}$ and Shimin $\mathrm{Hu}^{1}$ \\ ${ }^{1}$ Department of Hematopathology, The University of Texas MD Anderson Cancer Center, Houston, TX, USA \\ and ${ }^{2}$ Department of Leukemia, The University of Texas MD Anderson Cancer Center, Houston, TX, USA
}

\begin{abstract}
Classical Philadelphia chromosome-negative myeloproliferative neoplasms are a group of closely related myeloid disorders with different histologic features and clinical presentations at an early stage, but all later develop into a similar fibrotic stage with variable risk of acute transformation. The significance of 3q26.2/EVI1 rearrangement has been well recognized in acute myeloid leukemia, myelodysplastic syndrome, and chronic myeloid leukemia. However, the clinical importance of 3q26.2/EVI1 rearrangement in classical Philadelphia chromosome-negative myeloproliferative neoplasms is unknown. Here we reported 15 patients with classical Philadelphia chromosome-negative myeloproliferative neoplasms showing 3q26.2 rearrangement, including inv (3)(q21q26.2) $(n=6), \mathrm{t}(3 ; 21)(\mathrm{q} 26.2 ; \mathrm{q} 22)(n=4), \mathrm{t}(3 ; 3)(\mathrm{q} 21 ; \mathrm{q} 26.2)(n=3)$, inv(3)(q13.3q26.2)( $n=1)$, and $\mathrm{t}(3 ; 12)(\mathrm{q} 26.2$; p13) $(n=1)$. In addition to 3q26.2 rearrangement, 9 of 15 cases had other concurrent karyotypical abnormalities, including $-7 / 7 q-$ and $-5 / 5 q-$. There were 8 men and 7 women with a median age of 59 years (range, 35-79 years) at initial diagnosis of myeloproliferative neoplasms: 8 patients had primary myelofibrosis, 4 had polycythemia vera, and 3 had essential thrombocythemia. JAK2 V617F mutation was detected in 8/14 patients, including 4/4 with polycythemia vera. The median interval from the initial diagnosis of myeloproliferative neoplasms to the detection of 3q26.2 rearrangement was 44 months (range, 1-219 months). At time of emergence of 3q26.2 rearrangement, 11 patients were in blast phase and 2 patients had increased blasts (6-19\%). Dyspoiesis, predominantly in megakaryocytes, were detected in all patients with adequate specimens at time of 3q26.2 rearrangement. Following 3q26.2 rearrangement, 12 patients received chemotherapy, but none of them achieved complete remission. Of 14 patients with follow-up information, all died with a median overall survival time of only 3 months (range 0-14 months) after the emergence of 3q26.2 rearrangement. In summary, 3q26.2 rearrangement in classical Philadelphia chromosome-negative myeloproliferative neoplasms is associated with other concurrent cytogenetic abnormalities, a rapid disease progression and blast transformation, a poor response to chemotherapy and a dismal prognosis.
\end{abstract}

Modern Pathology (2017) 30, 940-951; doi:10.1038/modpathol.2017.19; published online 24 March 2017

Classical Philadelphia chromosome-negative myeloproliferative neoplasms are a group of clonal hematopoietic disorders characterized by increased proliferation of one or more hematopoietic cell lineages, and include three major subgroups: polycythemia vera, essential thrombocythemia, and

Correspondence: Dr S Hu, MD, PhD, Department of Hematopathology, The University of Texas MD Anderson Cancer Center, 1515 Holcombe Boulevard, Houston, TX 77030, USA.

E-mail: shu1@mdanderson.org

Received 22 November 2016; revised 20 February 2017; accepted 21 February 2017; published online 24 March 2017 primary myelofibrosis. ${ }^{1-4}$ Each subgroup has its own characteristic morphologic features and clinical presentations. At the early stage, these neoplasms are characterized by effective hematopoiesis and cytosis. But all have the tendency to undergo a stepwise progression over time that results in bone marrow failure due to myelofibrosis, ineffective hematopoiesis, or transformation to acute leukemia. These changes are often associated with genetic evolution. ${ }^{5,6}$

Molecular mutations have been investigated to explain the pathogenesis of Philadelphia chromosomenegative myeloproliferative neoplasms. Acquired 
somatic mutations of $J A K 2, M P L$, and $C A L R$ are critical events in the initial pathogenesis of many cases of Philadelphia chromosome-negative myeloproliferative neoplasms. ${ }^{2,7,8}$ However, these mutations do not provide a complete explanation for disease progression. Cytogenetic abnormalities are an essential part of prognostic systems, and their prognostic relevance has been well recognized in myeloid malignancies, including acute myeloid leukemia, myelodysplastic syndrome, and chronic myeloid leukemia. ${ }^{9-12}$ Although most chromosomal abnormalities observed in Philadelphia chromosome-negative myeloproliferative neoplasms are nonspecific, cytogenetic analysis is still recommended as part of the diagnostic work-up. Cytogenetic abnormalities occur in up to $30 \%$ patients of primary myelofibrosis, $20 \%$ of polycythemia vera, and $5-10 \%$ of essential thrombocythemia. ${ }^{13}$ The potential role of cytogenetic alterations in the progression of Philadelphia chromosome-negative myeloproliferative neoplasms is unknown.

The MDS1 and ectotropic virus integration site 1 (EVI1) complex (MECOM) locus is located in chromosomal band 3q26.2, giving rise to two major mRNA and protein species, EVI1 and MDS1. Chromosomal rearrangements involving the 3q26.2 locus reported previously include inv(3)(q21q26.2), $\mathrm{t}(3 ; 3)(\mathrm{q} 21 ; \mathrm{q} 26.2), \quad \mathrm{t}(3 ; 21)(\mathrm{q} 26.2 ; \mathrm{q} 22), \quad \mathrm{t}(3 ; 12)(\mathrm{q} 26.2 ;$ p13), and other rare reciprocal translocations involving 3q26.2, and have been recognized in acute myeloid leukemia, myelodysplasia syndrome, and accelerated and blast phases of chronic myeloid leukemia. These chromosomal aberrations involve the EVI1 oncogene, which encodes an essential transcription factor for the regulation of selfrenewal of hematopoietic stem cells. ${ }^{14,15}$ Acute myeloid leukemia with $\operatorname{inv}(3) / t(3 ; 3)$ is known to be associated with atypical megakaryocytes, multilineage dysplasia, and an unfavorable prognosis, and is listed among acute myeloid leukemia with recurrent cytogenetic abnormalities in current WHO classification. ${ }^{3,10,16}$ Myelodysplastic syndrome patients with inv(3;3)/t(3;3) have a poor prognosis similar to acute myeloid leukemia patients with inv $(3 ; 3) / t(3 ; 3)$, regardless of the blast percentage. ${ }^{9}$ Similar to $\operatorname{inv}(3 ; 3) / t(3 ; 3), t(3 ; 21)$ is also associated with a poor clinical outcome in acute myeloid leukemia and myelodysplastic syndrome. A recent large study based on 2013 chronic myeloid leukemia patients showed that chronic myeloid leukemia patients with 3q26.2 rearrangement have a minimal response to tyrosine kinase inhibitor therapy, a high rate of blastic transformation, and a poor overall survival. ${ }^{11,12}$

To date, few, if any, studies have focused on 3q26.2 rearrangement at the cytogenetic level in patients with classical Philadelphia chromosomenegative myeloproliferative neoplasms. In this study, we described the clinicopathologic features of 15 patients of classical Philadelphia chromosomenegative myeloproliferative neoplasms with 3 q26.2 rearrangement. Similar to the role of 3q26.2 rearrangement in the progression of chronic myeloid leukemia, our data demonstrated that $3 \mathrm{q} 26.2$ arrangement in classical Philadelphia chromosomenegative myeloproliferative neoplasms is associated with a rapid disease progression, a treatment resistance and a dismal outcome.

\section{Materials and methods}

\section{Patient Selection}

Cases of classical Philadelphia chromosomenegative myeloproliferative neoplasms diagnosed in our institution since 1998 through 2015 were reviewed to identify those with 3 q26.2 rearrangement. Patients' clinical information including treatment regimens, disease progression, and follow-up data were obtained. This study was approved by the Institutional Review Board at the University of Texas MD Anderson Cancer Center.

\section{Morphologic Studies}

Bone marrow core biopsies were prepared following formalin fixation and decalcification, and were stained with hematoxylin and eosin. Bone marrow aspirate and touch imprint smears as well as peripheral blood smear were stained with WrightGiemsa stain. Bone marrow differential count was performed by counting 500 nucleated cells from aspirate smears and/or 300 nucleated cells from touch imprint smears. Immunohistochemistry and special stains, including reticulin and trichrome stains, were performed on the biopsy specimens.

\section{Immunohistochemistry and Flow Cytometric Analyses}

Immunohistochemical studies were performed using formalin-fixed paraffin-embedded tissue sections according to standard protocols. The antibodies used included CD34 (1:40; BD Biosciences, San Jose, CA, USA), CD61 (1:100; Cell Marque, Rocklin, CA, USA), CD117 (1:100), myeloperoxidase (1:6000), and TdT (1:50; Dako, Carpinteria, CA, USA). Flow cytometry immunophenotypic analysis was performed using standard multicolor analysis which evolved substantially during the study interval. The panel of antibodies included reagents specific for CD2, CD3, CD4, CD5, CD7, CD10, CD11c, CD13, CD15, CD19, CD20, CD22, CD25, CD30, CD34, CD38, CD41, CD45, CD61, CD79b, CD117, CD123, and HLA-DR (Becton-Dickinson, Biosciences, San Jose, CA, USA).

\section{Cytogenetic Studies}

Conventional chromosomal analysis was performed on G-banded metaphase cells prepared from unstimulated bone marrow aspirate cultures ( 24 and $48 \mathrm{~h}$ ) using standard methods. The karyotype was reported according to the current International System for 
Human Cytogenetic Nomenclature. ${ }^{17}$ Florescence in situ hybridization (FISH) was performed on freshly harvested aspirate smears or cultured bone marrow cells according to the standard protocol. The KREATECH MECOM (EVI1) (SO/SG) FISH probe (Leica Biosystems, Buffalo Grove, IL, USA) was used in our present study. A total of 200 interphase nuclei were analyzed.

\section{Molecular Studies}

All molecular studies were performed on the bone marrow aspirate samples. Quantitative real-time reverse transcriptase polymerase chain reaction (PCR) analysis was performed for the BCR-ABL1 fusion transcripts e13a2 (b2a2), e14a2 (b3a2) and e1a2. CEBPA (CCAAT/enhancing binding proteinalpha) mutation was detected by using PCR-based DNA sequencing analysis. In recent cases, nextgeneration sequencing-based analysis for the detection of somatic mutations in the coding sequence of a total of 28 genes (including DNMT3A, JAK2, NPM1, FLT3, MPL, NRAS, KRAS, KIT, IDH1, IDH2, and TP53) was performed on the DNA extracted from the aspirate samples.

\section{International Prognostic Index}

Dynamic International Prognostic Scoring System (DIPSS) and DIPSS-plus were used to estimate prognosis in patients with Philadelphia chromosome-negative myeloproliferative neoplasms following the emergence of 3q26.2 rearrangement. The clinical characteristics in the DIPSS model include age, constitutional symptoms, white blood cell count, hemoglobin, and blast count in peripheral blood. Besides DIPSS risk factors, the DIPSS-plus score also includes karyotype, transfusion dependency, and platelet count. In these models, the prognosis is based on a 0-6-point scoring system. The DIPSS-plus score and its corresponding prognosis are listed below: score 0: low risk, estimated median survival 180 months; score 1: intermediate-1 risk, estimated median survival 80 months; score 2-3: intermediate-2 risk, estimated median survival 35 months; score 4 or more: high risk, estimated median survival 16 months. ${ }^{18}$

\section{Results}

\section{Clinical Characteristics}

A total of 1842 patients diagnosed with classical Philadelphia chromosome-negative myeloproliferative neoplasms since 1998 through 2015 had karyotyping information available, and $15(0.8 \%)$ of them were identified to have 3q26.2 rearrangement. There were 8 men and 7 women with a median age of 59 years (range, 35-79 years) at the time of initial diagnosis of myeloproliferative neoplasms. Eight patients had a diagnosis of primary myelofibrosis, 4 had polycythemia vera, and 3 had essential thrombocythemia. 3q26.2 rearrangement developed a median time of 44 months (range 1-219 months) after the initial diagnosis of myeloproliferative neoplasms (Table 1). The median interval for the emergence of 3q26.2 rearrangement in primary myelofibrosis, polycythemia vera, and essential thrombocythemia was 30,96 , and 61 months, respectively.

\section{Morphologic and Immunophenotypic Findings}

At the time of detection of 3q26.2 rearrangement, dysplasia in one or multi-lineages was detected in all patients with bone marrow showing adequate cellularity for morphologic evaluation: dysgranulopoiesis in 6/14 (43\%), dyserythropoiesis in 8/12 (67\%), and dysmegakaryopoiesis in $13 / 13(100 \%)$ patients (Table 2). Megakaryocytes were increased in 10 patients. Dysmegakaryopoiesis showed diverse morphology including small hyperchromatic, hypolobated/monolobated, or large atypical megakaryocytes (Figure 1). The number and morphology of megakaryocytes were noted to change with the disease progression. Reticulin and trichrome stains were performed on bone marrow biopsy specimens in 14 patients: 6 patients had MF-3, 7 had MF-2, and 1 had MF-1. As an example, the histological findings of case \#12 before and after 3q26.2 emergence are illustrated in Figure 2.

At the time of detection of 3q26.2 rearrangement, $13(87 \%)$ patients had increased blast count, ranging from $8 \%$ to $92 \% ; 11 / 15(73 \%)$ had blast phase ( $\geq 20 \%$ blasts in bone marrow or peripheral blood), 1 had accelerated phase (patient \#8, 14\% blasts), and 1 (patient \#2) had $8 \%$ blasts in the bone marrow. Of note, patient \#2 developed blast crisis 5 months after the emergence of $3 q 26.2$ rearrangement (53\% blasts in bone marrow; $44 \%$ in peripheral blood). Of the two patients (\#9 and \#15) who had no increase in blasts, patient \#9 had an accelerated phase (12\% blasts in bone marrow) 12 months after the emergence of 3q26.2 rearrangement.

Of the 13 patients with increased blasts, blasts in 12 patients (except patient \#1) had myeloid phenotype. Patient \#1 had one subset of blasts with erythroid differentiation and another subset with megakaryocytic differentiation. Five (5/12) patients had blasts with other lineage differentiation. For example, Patient \#3 showed blasts with abundant CD61 expression indicating megakaryocytic differentiation. Patients \#4, 7, 11, and 12 had blasts with CD4, CD14, and/or CD64 expression indicating monocytic differentiation. Interestingly, case \#4 showed two blast populations, a predominant myeloblast population and a small population of blasts with aberrant expression of CD19 and CD22, indicating B-lymphoid lineage (Table 2). 


\begin{tabular}{|c|c|c|c|c|c|c|c|c|c|c|}
\hline Patient \# & Gender & $\begin{array}{l}\text { Age of } \\
\text { Dx } \\
\text { (years) }\end{array}$ & MPN subgroup & $\begin{array}{l}\text { Latency of } 3 q 26.2 \\
\text { rearrangement } \\
\text { (months) }\end{array}$ & $\begin{array}{l}\text { Treatment before } \\
3 q 26.2 \\
\text { rearrangement }\end{array}$ & $\begin{array}{l}\text { Treatment after } 3 q 26.2 \\
\text { rearrangement }\end{array}$ & $\begin{array}{l}\text { Treatment } \\
\text { response }\end{array}$ & Status & $\begin{array}{c}\text { Overall } \\
\text { survival after } \\
\text { initial Dx } \\
\text { (months) }\end{array}$ & $\begin{array}{l}\text { Overall survival after } \\
3 q 26.2 \text { rearrangement } \\
\text { (months) }\end{array}$ \\
\hline 1 & $\mathrm{~F}$ & 60 & $\begin{array}{l}\text { Primary } \\
\text { myelofibrosis }\end{array}$ & 35 & $\begin{array}{l}\text { Prednisone and } \\
\text { hydroxyurea }\end{array}$ & Unknown regimens & Unknown & Dead & 35 & $0<1$ \\
\hline 2 & $\mathrm{M}$ & 75 & $\begin{array}{l}\text { Primary } \\
\text { myelofibrosis }\end{array}$ & 1 & $\begin{array}{l}\text { Ruxolitinib and } \\
\text { azacytidine }\end{array}$ & $\begin{array}{l}\text { Cladribine, cytarabine, } \\
\text { decitabine }\end{array}$ & Relapsed & Dead & 11 & 10 \\
\hline 3 & M & 35 & $\begin{array}{l}\text { Primary } \\
\text { myelofibrosis }\end{array}$ & 200 & No treatment & $\begin{array}{l}\text { Idarubicin, cytarabine, } \\
\text { hydroxyurea }\end{array}$ & No response & Dead & 201 & 1 \\
\hline 4 & $\mathrm{M}$ & 38 & $\begin{array}{l}\text { Primary } \\
\text { myelofibrosis }\end{array}$ & 2 & $\begin{array}{l}\text { Interferon, } \\
\text { thalidomide, } \\
\text { hydroxyurea }\end{array}$ & Unknown regimens & Unknown & Dead & 7 & 5 \\
\hline 5 & M & 58 & $\begin{array}{l}\text { Primary } \\
\text { myelofibrosis }\end{array}$ & 145 & $\begin{array}{l}\text { Hydroxyurea, } \\
\text { steroids }\end{array}$ & $\begin{array}{l}\text { Omacetaxine and } \\
\text { cytarabine }\end{array}$ & No response & Dead & 148 & 3 \\
\hline 6 & $\mathrm{M}$ & 65 & $\begin{array}{l}\text { Primary } \\
\text { myelofibrosis }\end{array}$ & 26 & Hydroxyurea & Cytarabine and idarubicin & No response & Dead & 37 & 12 \\
\hline 7 & $\mathrm{M}$ & 62 & $\begin{array}{l}\text { Primary } \\
\text { myelofibrosis }\end{array}$ & 43 & $\begin{array}{l}\text { Hydroxyurea, and } \\
\text { growth factors }\end{array}$ & $\begin{array}{l}\text { Revlimid, prednisone, 5- } \\
\text { azacytidine, valproic acid, } \\
\text { and ATRA }\end{array}$ & No response & Dead & 47 & 4 \\
\hline 8 & $\mathrm{~F}$ & 71 & $\begin{array}{l}\text { Primary } \\
\text { myelofibrosis }\end{array}$ & 8 & $\begin{array}{l}\text { Transfusion, } \\
\text { danazol, and HDAC } \\
\text { inhibitor }\end{array}$ & Pracinostat, decitabine & No response & Dead & 18 & 3 \\
\hline 9 & $\mathrm{~F}$ & 71 & Polycythemia vera & 15 & $\begin{array}{l}\text { Hydroxyurea, and } \\
\text { aranesp }\end{array}$ & JAK2 inhibitor & $\begin{array}{l}\text { Partial } \\
\text { response }\end{array}$ & Dead & 17 & 2 \\
\hline 10 & $\mathrm{~F}$ & 44 & Polycythemia vera & 148 & $\begin{array}{l}\text { Hydroxyurea; } \\
\text { phlebotomy }\end{array}$ & $\begin{array}{l}\text { Fludarabine, cytarabine, } \\
\text { and JAK2 inhibitor }\end{array}$ & No response & Dead & 153 & 4 \\
\hline 11 & $\mathrm{M}$ & 57 & Polycythemia vera & 44 & $\begin{array}{l}\text { Phlebotomy and } \\
\text { hydroxyurea }\end{array}$ & Fludarabine and cytarabine & No response & Dead & 56 & 12 \\
\hline 12 & $\mathrm{~F}$ & 59 & Polycythemia vera & 219 & $\begin{array}{l}\text { phlebotomy, } \\
\text { hydroxyurea, and } \\
\text { vidaza }\end{array}$ & $\begin{array}{l}\text { Cladribine, cytarabine, } \\
\text { mitoxantrone, Vidaza }\end{array}$ & No response & Dead & 220 & $<1$ \\
\hline 13 & $\mathrm{~F}$ & 53 & $\begin{array}{l}\text { Essential } \\
\text { thrombocythemia }\end{array}$ & 152 & $\begin{array}{l}\text { Hydroxyurea and } \\
\text { procrit }\end{array}$ & $\begin{array}{l}\text { Idarubicin, cytarabine, and } \\
\text { methylprednisolone }\end{array}$ & $\begin{array}{l}\text { Partial } \\
\text { response }\end{array}$ & Dead & 153 & 1 \\
\hline 14 & $\mathrm{M}$ & 79 & $\begin{array}{l}\text { Essential } \\
\text { thrombocythemia }\end{array}$ & 44 & Hydroxyurea & Aurora kinase inhibitor & No response & Dead & 58 & 14 \\
\hline 15 & $\mathrm{~F}$ & 61 & $\begin{array}{l}\text { Essential } \\
\text { thrombocythemia }\end{array}$ & 61 & $\begin{array}{l}\text { Anagrelide and } \\
\text { hydroxyurea }\end{array}$ & Unknown regimens & Unknown & Unknown & NA & NA \\
\hline
\end{tabular}

Abbreviations: ATRA, all-trans-retinoic acid; Dx, diagnosis; NA, not applicable. 
Table 2 Morphologic and immunophenotypic findings in Philadelphia chromosome-negative myeloproliferative neoplasms with 3q26.2 rearrangement

\begin{tabular}{|c|c|c|c|c|c|c|}
\hline Patient \# & $M F$ & Granulocytes & Erythrocytes & Megakaryocytes & Blasts (\%, Interval) & Blast immunophenotype \\
\hline 1 & MF-3 & Markedly decreased & $\begin{array}{l}\text { Markedly increased, dysplastic, } \\
\text { and }<5 \% \text { ringed sideroblasts }\end{array}$ & Increased, dysplastic & $\begin{array}{c}11 \%, 0(\mathrm{BM}) 67 \% \\
(\mathrm{~PB})\end{array}$ & $\begin{array}{l}\text { One subset ( } 37 \% \text { ) expressing glycophorin A; another subset ( } 25 \% \text { ) } \\
\text { expressing CD41 and CD61 }\end{array}$ \\
\hline 2 & MF-2 & Unremarkable & Mild dysplastic & Increased, dysplastic & $53 \%, 5$ months & $\begin{array}{l}\text { Positive for CD13, CD15, CD33, CD34, CD38, CD45, CD56, CD117, } \\
\text { and CD123; negative for CD4 and CD14 }\end{array}$ \\
\hline 3 & MF-3 & Markedly decreased & Markedly decreased & Increased, dysplastic & $92 \%, 0$ & $\begin{array}{l}\text { Positive for CD13, CD34, CD61, and HLA-DR; Negative for CD3, CD7, } \\
\text { CD19, CD20, CD117, TdT, and glycophorin A }\end{array}$ \\
\hline 4 & MF-2 & Dysplastic & Dysplastic & Increased, dysplastic & $20 \%, 0$ & $\begin{array}{l}\text { Major myeloid blast population positive for CD13, CD14, CD33, } \\
\text { CD34 (small subset), CD38, CD41, CD49d, partial CD56, CD64, and } \\
\text { HLA-DR; negative for CD15, CD117, MPO, and T- and B-cell } \\
\text { markers. Minor B-lymphoblast population }(<10 \%) \text { positive for } \\
\text { CD19, CD22, and CD34; negative for CD10 }\end{array}$ \\
\hline 5 & MF-3 & $\begin{array}{l}\text { Too rare to assess } \\
\text { morphology }\end{array}$ & Too rare to assess morphology & Increased, dysplastic & $30 \%, 0$ & $\begin{array}{l}\text { Positive for CD13, CD33, CD34, CD38, CD117, and HLA-DR; negative } \\
\text { for CD2, CD7, CD14, CD19, CD36, CD64, and TdT }\end{array}$ \\
\hline 6 & MF-1 & Unremarkable & Mildly dysplastic & Increased, dysplastic & $30 \%, 0$ & $\begin{array}{l}\text { Positive for CD13, CD33, CD34, CD117, HLA-DR, and MPO (subset); } \\
\text { negative for CD14, CD15, CD41, CD56, CD64, MPO, TdT }\end{array}$ \\
\hline 7 & MF-2 & Dysplastic & Decreased & $\begin{array}{l}\text { Too rare to assess } \\
\text { morphology }\end{array}$ & $35 \%, 0$ & $\begin{array}{l}\text { Positive for CD13, CD14, CD15 (small subset), CD33, CD34, CD38, } \\
\text { CD56 (partial), CD64, CD117, HLA-DR (subset), and MPO (partial); } \\
\text { negative for CD3, CD19, CD41, and TdT }\end{array}$ \\
\hline 8 & MF-2 & $\begin{array}{l}\text { Decreased, unremarkable } \\
\text { morphology }\end{array}$ & Dysplastic & Decreased, dysplastic & $14 \%, 0$ & $\begin{array}{l}\text { Positive for CD5, CD13, CD33, CD34, CD38, CD117, and HLA-DR; } \\
\text { negative for CD3, CD7, CD19, CD20, and TdT }\end{array}$ \\
\hline 9 & MF-3 & Unremarkable & Rare & No intact forms identified & $12 \%, 12$ months & NA \\
\hline 10 & MF-3 & Decreased, dysplastic & Markedly decreased & $\begin{array}{l}\text { Patchy distribution; } \\
\text { dysplastic }\end{array}$ & $40 \%, 0$ & $\begin{array}{l}\text { Positive for CD7, CD13, CD33, CD34, CD38, HLA-DR, and MPO } \\
\text { (subset); negative for CD14, CD15, CD41, CD56, CD64, TdT, and } \\
\text { other T- or B-cell markers }\end{array}$ \\
\hline 11 & $\mathrm{NA}$ & Left shift maturation & Mildly dysplastic & Increased, dysplastic & $29 \%, 0$ & $\begin{array}{l}\text { One population (18\%) positive for CD13, CD34, CD38, CD117, and } \\
\text { HLA-DR; another population ( } 66 \% \text { ) positive for CD14, CD33, CD38, } \\
\text { CD56, CD64, and HLA-DR. Both populations negative for CD3 and } \\
\text { CD19. }\end{array}$ \\
\hline 12 & MF-3 & Decreased & Decreased markedly & Decreased, dysplastic & $65 \%, 0$ & $\begin{array}{l}\text { Positive for CD4 (partial/dim), CD7(subset), CD13, CD33(80\%), } \\
\text { CD34, CD38, CD45 (dim), CD64 (partial), CD117, and HLA-DR; } \\
\text { negative for CD2, CD5, CD14, CD15, CD19, CD22, CD36, CD56, and } \\
\text { CD123 }\end{array}$ \\
\hline 13 & MF-2 & Mildly dysplastic & Too rare to assess morphology & Increased, dysplastic & $32 \%, 0$ & $\begin{array}{l}\text { Positive for CD13, CD33, CD34, CD117, and HLA-DR; negative for } \\
\text { CD2, CD3, CD19, CD20, CD64, and TdT }\end{array}$ \\
\hline 14 & MF-2 & $\begin{array}{l}\text { Left-shifted, mildly } \\
\text { dysplastic }\end{array}$ & Mildly dysplastic & Increased, dysplastic & $30 \%, 0$ & $\begin{array}{l}\text { Positive for CD7, CD10 (partial), CD13, CD15 (partial), CD33, CD34, } \\
\text { CD38, CD117, and MPO (partial); negative for CD2, CD3, CD5, CD14, } \\
\text { CD19, CD20, CD56, CD64, glycophorin A, and TdT }\end{array}$ \\
\hline 15 & MF-2 & Mildly dysplastic & Mildly dysplastic & Increased, dysplastic & $4 \%, N A$ & NA \\
\hline
\end{tabular}

BM, bone marrow; MF, myelofibrosis; MPO, myeloperoxidase; NA, not applicable; PB, peripheral blood. Interval represents the period between the emergence of 3q26.2 rearrangement and blast phase or any increase in blast count. 


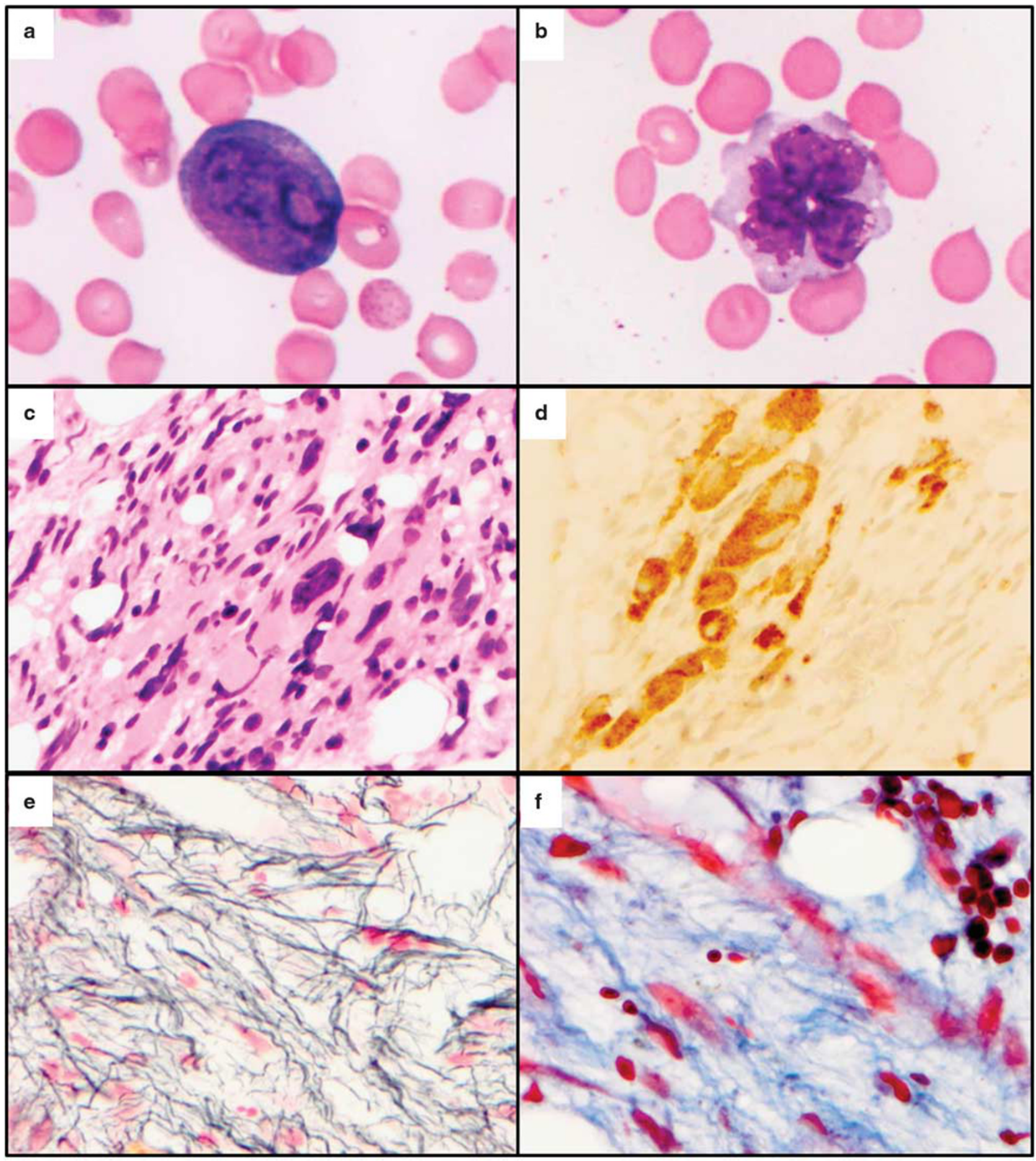

Figure 1 Morphologic findings at emergence of 3q26.2 rearrangement in a patient (\#8) with primary myelofibrosis. (a) Morphology of myeloblasts in peripheral blood (×1000). (b) Morphology of monocytes in peripheral blood $(\times 1000)$. (c) H\&E stained bone marrow biopsy $(\times 500)$ showing fibrosis and atypical megakaryocytes. (d) CD61 immunohistochemical stain highlighting atypical megakaryocytes $(\times 500)$. (e) Reticulin stain $(\times 1000)$ showing diffuse and dense increase in reticulin fibers and extensive intersections. (f) Trichrome stain highlighting course collagen fibers $(\times 1000)$.

\section{Cytogenetics and Molecular Findings}

Rearrangements involving 3q26.2 locus were diverse in this cohort and included inv(3)(q21q26.2) $(n=6)$, $\mathrm{t}(3 ; 21)(\mathrm{q} 26.2 ; \mathrm{q} 22)(n=4), \mathrm{t}(3 ; 3)(\mathrm{q} 21 ; \mathrm{q} 26.2)(n=3)$, inv (3)(q13.3q26.2) $(n=1)$, and $\mathrm{t}(3 ; 12)(\mathrm{q} 26.2 ; \mathrm{p} 13)(n=1$;
Table 3). The 3q26.2/EVI1 rearrangements were confirmed by FISH analyses in $4 / 4$ patients (patients \#2, 9, 11, and 12; Figure 3). Nine of 15 patients had additional karyotypical abnormalities in the same clone of 3q26.2 rearragnements, including -7/7q- in 6 patients, $-5 / 5 q-$ in 5 patients, and -17 in 3 patients. 


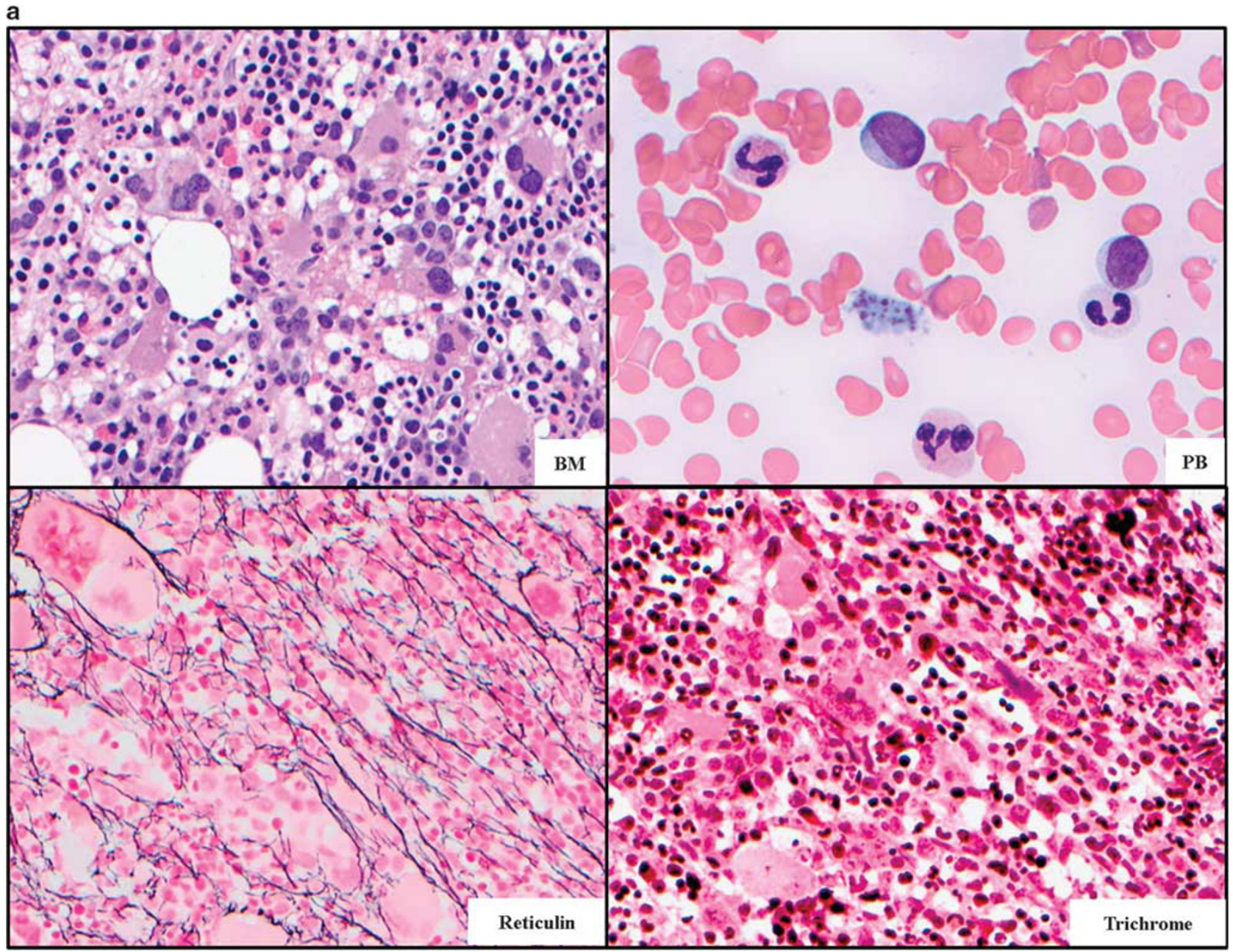

Figure 2 Morphologic findings before and at emergence of 3q26.2 rearrangement in a patient (\#12) with polycythemia vera. (a) Before the emergence of 3q26.2 rearrangement $(\times 500)$. H\&E stained bone marrow biopsy showing hypercellular marrow with increased atypical megakaryocytes. Wright-Giemsa stained aspirate smear showing morphology of myeloblasts. Reticulin stain showing a loose network of reticulin fibers. Trichrome stain showing no stainable collagen fibers. (b) At emergence of 3q26.2 rearrangement $(\times 500)$. H\&E stained bone marrow biopsy showing hypercellular marrow with numerous blasts. Wright-Giemsa stained aspirate smear showing blasts and dysplastic granulocyte. Reticulin stain showing diffuse and dense increase in reticulin fibers. Trichrome stain revealing course collagen fibers.

Patient \#2 had a karyotype of 46,XY,t(3;3)(q21;q26.2) [2]/46,XY,add(17)(p11.2)[2]/46,XY[4], in which add (17)(p11.2) was present in a different subclone without $\mathrm{t}(3 ; 3)$. Patient \#7 had additional inv(9) (p11q12) in the clone of $\mathrm{t}(3 ; 21)$, which is a chromosomal polypormorphism with no known clinical significance.

JAK2 mutation analysis was performed in 14 patients, and was positive in 8 patients, including $4 / 4(100 \%)$ patients with polycythemia vera, $2 / 3$ $(68 \%)$ patients with essential thrombocythemia, and $2 / 7(29 \%)$ patients with primary myelofibrosis. Patient \#12 had TP53 and DNMT3A mutations in addition to JAK2 mutation. FLT3 mutation was positive in 1 patient with primary myelofibrosis (patient \#6). BCR-ABL1 was negative in $8 / 8$ patients, and $R A S$ mutation was negative in 6/6 patients.

\section{Treatment and Outcome}

Depending on the type of myeloproliferative neoplasms at initial diagnosis, patients received different treatment regimens. All patients except patient \#3 received treatment before the detection of 3q26.2 rearrangement, including hydroxyurea in 12 patients and phlebotomies in 3 patients with polycythemia vera. After 3q26.2 rearrangement was detected, 12 patients received chemo- or targeted therapy, and in the remaining 3 patients, the treatment regimens were not known. The detailed treatment and followup are illustrated in Table 1. Of the 12 patients who received known therapy regimens after the emergence of 3q26.2 rearrangement, 9 patients (patients $\# 3,5,6,7,8,10,11,12$, and 14) had no response to treatment. The other 3 patients (patients \#2, 9, and 13) had responses to some degree or relapse but similar poor outcome. Patient \#2 received cladribine 


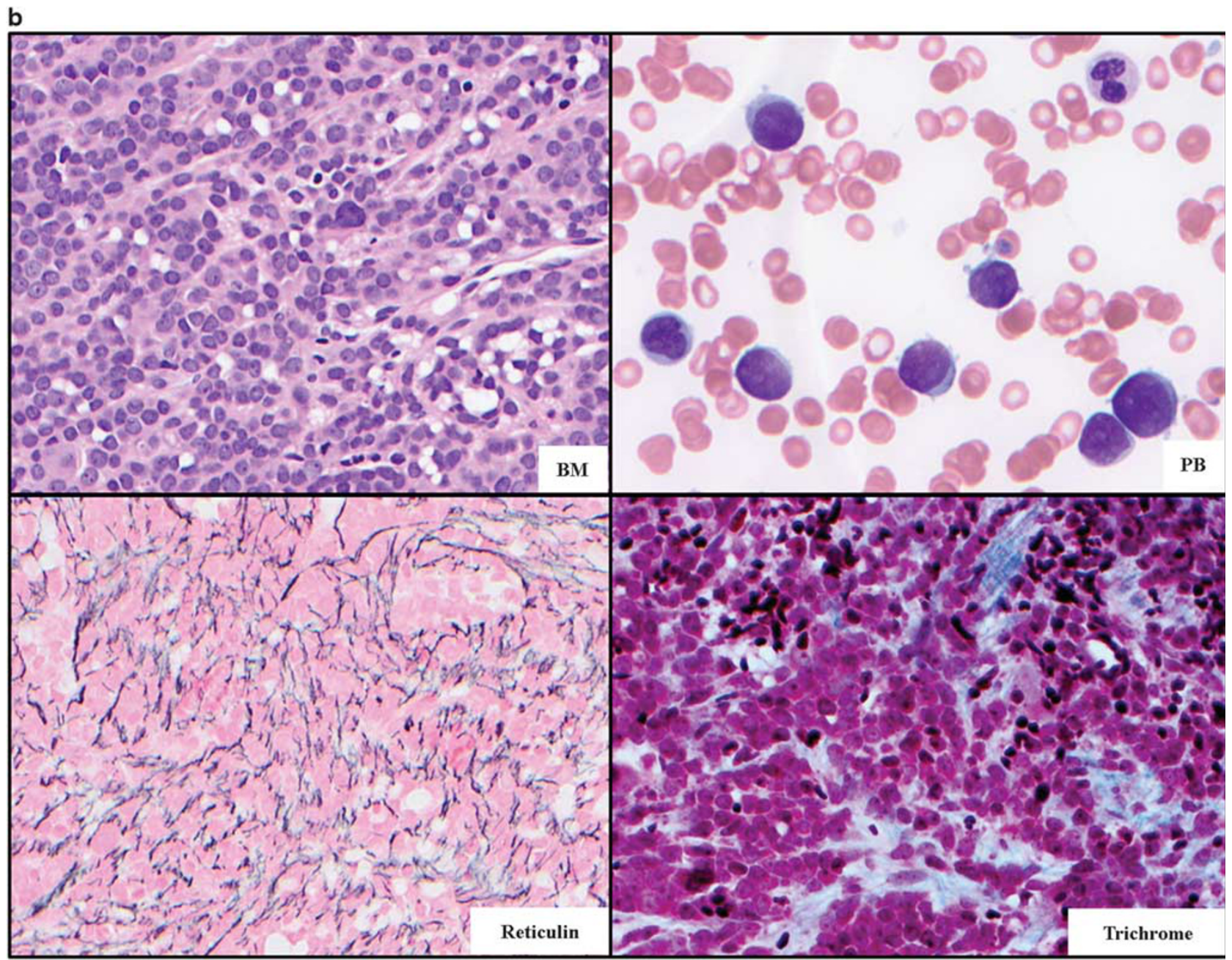

Figure 2 Continued.

plus low-dose ara-C alternating with decitabine for four cycles and achieved morphologic remission, but he developed relapsed disease soon and expired 10 months after 3q26.2 rearrangement was detected. Patient \#9 received lestaurtinib, a multitargeted tyrosine kinase inhibitor, and showed significant reduction of splenomegaly and platelet counts, as well as reduction in her transfusion dependence, but she developed pancytopenia and expired 2 months later. Patient \#13 received idarubicin, cytarabine and methylprednisone therapy and initially had significant reduction of blast count, but she developed pancytopenia and alveolar hemorrhage. She had relapsed disease and multi-organ failure and expired one month following $3 q 26.2$ rearrangement.

Of 14 patients with follow-up information, none of the patients survived progressive disease and were eligible for stem cell transplantation. The median overall survival after the initial diagnosis of myeloproliferative neoplasms was 51 months (range, 7-220 months). The median survival times for primary myelofibrosis, polycythemia vera and essential thrombocythemia subgroups were 36, 104, and 106 months, respectively. Following 3q26.2 rearrangement, the patients had a median overall survival of only 3 months (range, $0-14$ months). It seems that additional karyotypical abnormalities besides 3q26.2 rearrangement may have adverse impact on patient survival. Those with additional karyotypical abnormalities in the same clone of 3q26.2 rearrangement had a worse survival than those without (median: 3 months vs 7 months) although no statistical significance was reached $(P=0.25)$. There was statistically significant difference in the overall survival between patients with and without -17 (median: 1 month vs 4 months, $P=0.04$ ). These three patients with -17 had a highly complex karyotype.

\section{Prognosis in DIPSS and DIPSS-Plus Models}

Prognostic scores were calculated in this study cohort by using DIPSS and DIPSS-plus models. Application of the DIPSS resulted in categorization 
Table 3 Cytogenetic and molecular findings in Philadelphia chromosome-negative myeloproliferative neoplasms with 3q26.2 rearrangement

\begin{tabular}{|c|c|c|c|}
\hline Patient \# & Molecular findings & $3 q 26.2$ Group & Karyotype at time of 3q26.2 rearrangement \\
\hline 1 & JAK2 not examined & $\mathrm{t}(3 ; 3)$ & $46, X X, t(3 ; 3)(q 21 ; q 26.2)[18] / 45$, idem,-6,-7,+22[2] \\
\hline 2 & $J A K 2(+)$ & $t(3 ; 3)$ & 46,XY,t(3;3)(q21;q26.2)[2]/46,XY,add(17)(p11.2)[2]/46,XY[4] \\
\hline 3 & $J A K 2(-)$ & $\operatorname{inv}(3)$ & 46,XY,inv(3)(q21q26.2)[20] \\
\hline 4 & $J A K 2(-)$ & $\mathrm{t}(3 ; 21)$ & $\begin{array}{l}46, \mathrm{XY}, \mathrm{t}(3 ; 21)(\mathrm{q} 26.2 ; \mathrm{q} 22), \mathrm{add}(8)(\mathrm{q} 24.3), \mathrm{t}(8 ; 13)(\mathrm{p} 10 ; \mathrm{p} 10), \operatorname{del}(15) \\
(\mathrm{q} 24 \mathrm{q} 26.3)[20]\end{array}$ \\
\hline 5 & $J A K 2(-)$ & $\mathrm{t}(3 ; 21)$ & $\begin{array}{l}47, X Y, t(3 ; 21)(q 26.2 ; q 22),-5, \operatorname{add}(6)(q 21),-7, \operatorname{del}(7)(q 22 q 34),-17,-17,-20,-22, \\
+7 \operatorname{mar}[14] / 46, X Y[6]\end{array}$ \\
\hline 6 & JAK2(-), FLT3(+) & $\operatorname{inv}(3)$ & 46,XY,inv(3)(q21q26.2)[18]/46,idem,del(7)(q22q34)[2] \\
\hline 7 & $J A K 2(-)$ & $\mathrm{t}(3 ; 21)$ & $46, \mathrm{XY}, \mathrm{t}(3 ; 21)(\mathrm{q} 26.2 ; \mathrm{q} 22), \operatorname{inv}(9)(\mathrm{p} 11 \mathrm{q} 12)[19]$ \\
\hline 8 & $J A K 2(+)$ & $\operatorname{inv}(3)$ & 46,XX,inv(3)(q21q26.2),r(7)(p11.2q11.2)[19]/46,XX[1] \\
\hline 9 & $J A K 2(+)$ & $\mathrm{t}(3 ; 3)$ & $\begin{array}{l}\text { 46,XX,del(4)(q21q33)[7]/46,X,del(X)(q22q28)[6]/46,XX,t(3;3)(q21;q26.2), } \\
\operatorname{del}(5)(q 31 q 35), \operatorname{del}(6)(p 21.1 p 23), \operatorname{add}(20)(q 13.2)[2] / 46, X X[5]\end{array}$ \\
\hline 10 & $J A K 2(+)$ & $\operatorname{inv}(3)$ & $\begin{array}{l}\text { 46,XX,inv(3)(q21q26.2),del(5)(q31q35),add(7)(q36),del(9)(q22)[11]/46, } \\
\text { XX,del(5)(q31q35),der(6),t(1;6)(q21;p23)[5]/46,XX[4] }\end{array}$ \\
\hline 11 & $J A K 2(+)$ & $\operatorname{inv}(3)$ & $46, X Y, \operatorname{inv}(3)(q 21 q 26.2)[20]$ \\
\hline 12 & $\begin{array}{l}J A K 2(+), T P 53(+), D N M T 3 A \\
(+), C A L R / M P L(-)\end{array}$ & $\operatorname{inv}(3)(q 13.3 q 26.2)$ & $\begin{array}{l}\text { 43,X,-X,inv(3)(q13.3q26.2),del(5)(q22q35),add(6)(p21.1),-7,-17,-19,+mar } \\
\text { [8]/43,idem,del(11)(p11.2)[3]/44,idem,+mar[3]/44,idem,i(5)(q10),+mar } \\
{[2] / 46, X X[4]}\end{array}$ \\
\hline 13 & $J A K 2(-)$ & $\mathrm{t}(3 ; 21)$ & $\begin{array}{l}\text { 45,XX,del(3)(q11),t(3;21)(q26.2;q22),-4,del(5)(q13q33),-6,del(7)(q22q32),- } \\
\text { 16,-17,-18,+4mar[13]/46,idem,+21[2]/44 45,XX,t(3;21)(q26;q22),-5, del(7) } \\
\text { (q22q32),-16,-17,-18,+3-4mar[cp4] }\end{array}$ \\
\hline 14 & $J A K 2(+)$ & $\operatorname{inv}(3)$ & $46, X Y, \operatorname{inv}(3)(q 21 q 26.2)[20]$ \\
\hline 15 & $J A K 2(+)$ & $\mathrm{t}(3 ; 12)$ & $46, \mathrm{XX}, \mathrm{t}(3 ; 12)(\mathrm{q} 26.2 ; \mathrm{p} 13)[20]$ \\
\hline
\end{tabular}
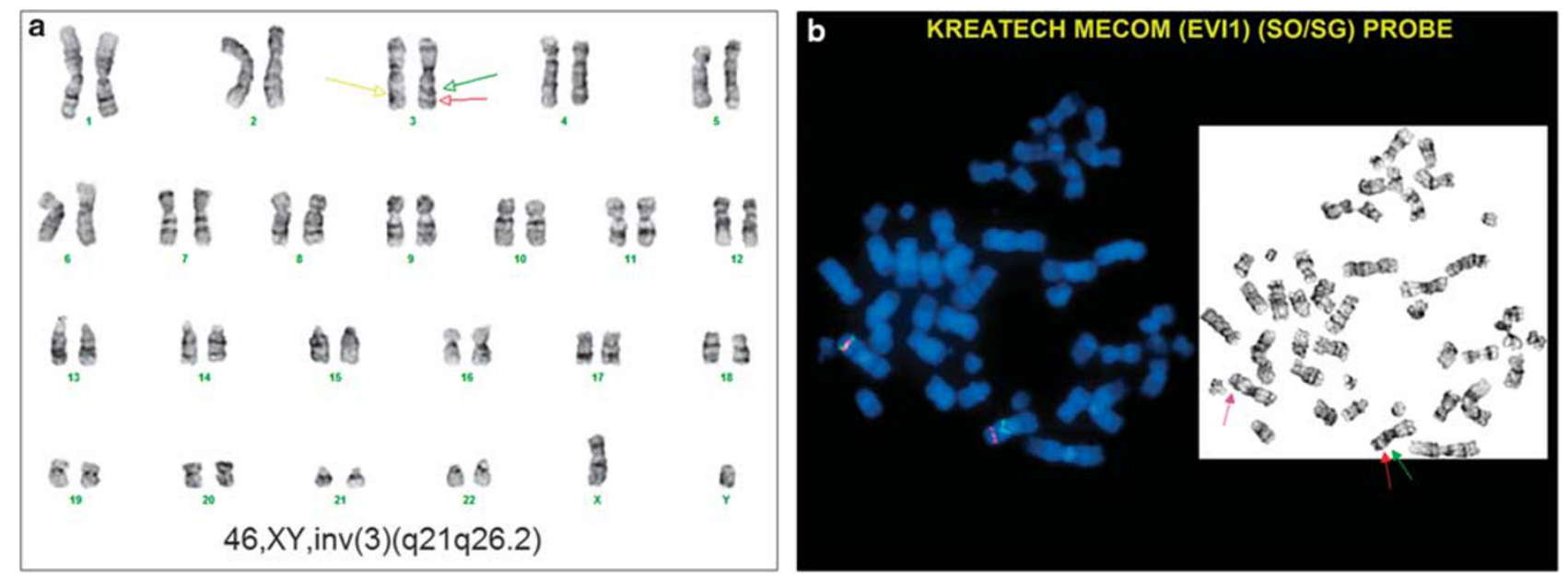

Figure 3 Cytogenetic findings at emergence of 3q26.2 rearrangement in a patient (\#11) with polycythemia vera. (a) G-banding chromosome analysis showing a karyotype of 46,XY,inv(3)(q21q26.2)[20]. (b) FISH using KREATECH MECOM (EVI1) (EO/SG) probe showing two separate signals (green and red) indicating EVI1 rearrangement.

of this cohort of patients into intermediate-1 $(n=4)$ and intermediate-2 ( $n=11$; Table 4$)$, with a predicted median overall survival of 35 months. By the DIPSSplus model, this cohort of patients were categorized into intermediate-2 risk $(n=2)$ and high-risk $(n=13)$, with a predicted median overall survival of 16 months. However, the actual median survival following 3q26.2 rearrangement in this cohort was only 3 months, markedly shorter than the overall survival predicted by both models, substantiating the dismal prognostic impact conferred by $3 \mathrm{q} 26.2$ rearrangement.

\section{Discussion}

In this study, we reported the clinicopathologic features of 15 patients with classical Philadelphia chromosome-negative myeloproliferative neoplasms showing 3q26.2 rearrangement. We found that $3 q 26.2$ rearrangement was associated with a dismal outcome. At the time of 3q26.2 rearrangement, the vast majority of patients developed blastic transformation. None of the patients responded to therapy and all had a poor survival following $3 q 26.2$ rearrangement. 


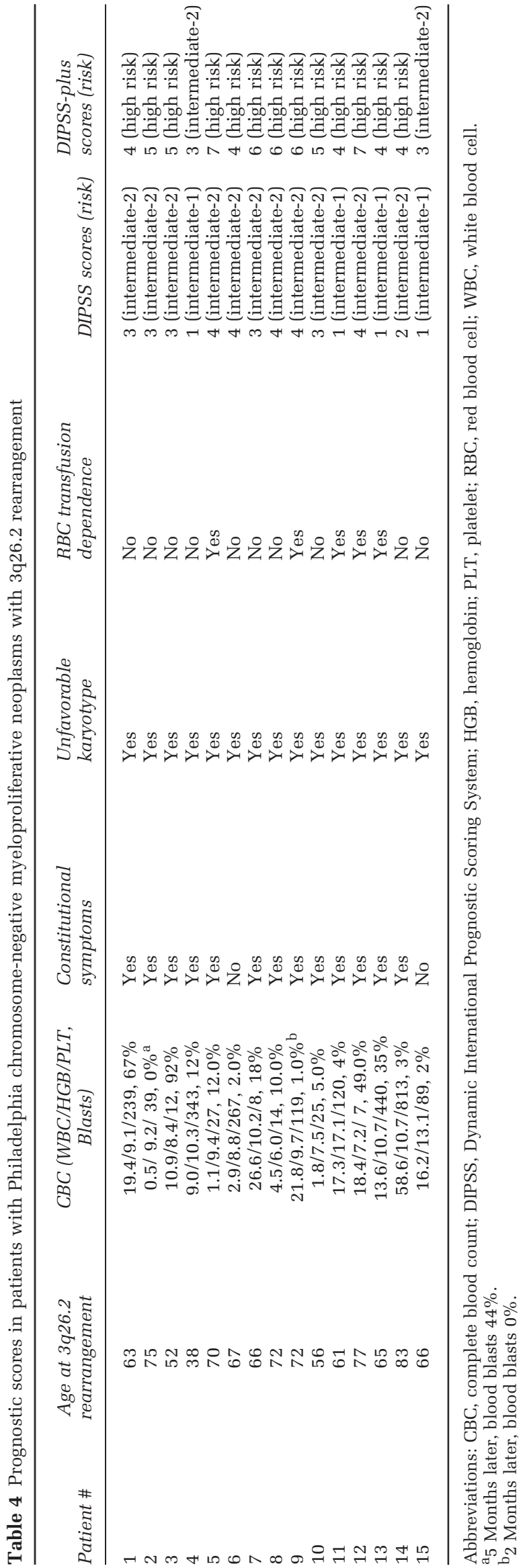

As mentioned above, 3q26.2 rearrangement has been identified in several types of myeloid neoplasms. Currently, 34 chromosomal rearrangements of 3q26.2 locus have been confirmed to have EVI1 involvement, including $\operatorname{inv}(3), \mathrm{t}(3 ; 3), \mathrm{t}(3 ; 21)$, and $\mathrm{t}(3 ; 12) .^{7,10,14}$ The $3 \mathrm{q} 26.2$ rearrangement leads to EVI1 dysregulation, which can be achieved through two different mechanisms. In in(3)(q21q26.2) and $t(3 ; 3)$ (q21;q26.2), inappropriate expression of EVI1 is likely caused by the juxtaposition of regulatory sequences (enhancers or promoters). In recent studies, using $\operatorname{inv}(3) / \mathrm{t}(3 ; 3)$ as a model, it is found that 3q26.2 rearrangement repositions a distal GATA2 enhancer to activate and cause deregulation of EVI1 gene. ${ }^{19,20}$ In $\mathrm{t}(3 ; 21)(\mathrm{q} 26.2 ; \mathrm{q} 22)$ and $\mathrm{t}(3 ; 12)$ (q26.2;p13), EVI1 gene fuses with RUNX1 and ETV6, respectively, resulting in the formation of chimeric fusion transcripts. ${ }^{10,21-23}$

Similar to patients with other types of myeloid neoplasms with 3q26.2 rearrangement, patients with classical Philadelphia chromosome-negative myeloproliferative neoplasms showing 3q26.2 rearrangement have a poor outcome. The presence of 3q26.2 rearrangement in different types of myeloid neoplasms suggests a common pathogenic pathway leading to disease progression. This concept was previously dubbed as '3q26.2 syndrome,' which is associated with normal or elevated platelet counts with marked abnormalities of megakaryopoiesis and involvement of multiple hematopoietic lineages. ${ }^{16,24}$ Based on the studies of EVI1 in myeloid malignancies, the development of ' $3 q 26.2$ syndrome' can be explained by a theoretical 'two-hit' process. The first hit is the primary neoplastic driver aberrations, transforming non-malignant cells to clonal neoplastic cells. In classical Philadelphia chromosomenegative myeloproliferative neoplasms, the molecular alterations including mutations of JAK2, MPL, and $C A L R$ can be the driver events. As the clonal progeny expands and disease burden increases, more mutations may occur, resulting in additional cytogenetic alterations that can be defined as 'second hit.' This second hit can produce an aggressive subclone with survival advantage and resistance to previous therapy. The event of 3q26.2 rearrangement can be regarded as a second hit of myeloid neoplasms.

The bone marrow of myelodysplastic syndrome/ acute myeloid leukemia patients with 3q26.2 rearrangement has characteristic dysmegakaryopoiesis including increased small monolobated or hypolobated megakaryocytes. ${ }^{14}$ However, in some cases of our study, the morphologic features of megakaryocytes were different from the characteristic dysmegakaryopoiesis in myelodysplastic syndrome/acute myeloid leukemia. Instead, they may show small monolobated morphology typical for myelodysplastic syndrome or large atypical megakaryocytes typical for myeloproliferative neoplasms. One potential explanation for the discrepancy is that some megakaryocytes retain their atypical morphology of 
myeloproliferative neoplasms after the emergence of 3q26.2 rearrangement.

Like patients with other myeloid neoplasms harboring 3q26.2 rearrangement, patients with classical Philadelphia chromosome-negative myeloproliferative neoplasms in our study were resistant to conventional chemotherapy and had a short overall survival. There is no consensus on the best therapy for these patients. Stem cell transplantation is thought to be the only potential therapy to cure the diseases. $^{25,26}$ However, no patients in our study survived long enough to receive stem cell transplantation due to the rapid disease deterioration. Search for new potential therapies is warranted for these patients. In theory, targeted therapies that block EVI1 functions may prevent the disease progression. In addition, mutations in RAS signaling pathway (NRAS, KRAS, PTPN11, and NF1) were identified in EVI1-rearranged acute myeloid leukemia. Groschel, et $a l^{22,27}$ found high rates of mutations in RAS signaling pathway (NRAS, KRAS and NF1) in acute myeloid leukemia, blast phase of chronic myeloid leukemia and myelodysplastic syndrome patients with 3q26.2 rearrangement. Thus, it is reasonable to speculate that novel drugs interfering with RAS signaling might be effective treating these patients resistant to the conventional chemotherapy. ${ }^{22,27,28}$

With regard to the prognostic models for myeloproliferative neoplasms, several prognostic models have been developed to predict the outcome of myelofibrosis since the first publication of the IPSS in $2009 .{ }^{29}$ In our study, we did not use the IPSS score for patients with 3q26.2 rearrangement because the IPSS can be used only at the time of initial diagnosis of myeloproliferative neoplasms. In contrast, DIPSS and DIPSS-plus can be used at any given time point during the disease course of myelofibrosis. ${ }^{5,18,30}$ The actual overall survival after the emergence of 3q26.2 rearrangement in our study is markedly shorter than the expected overall survival predicted by DIPSS. This indicates that the DIPSS score underestimates the poor prognosis in these patients. The DIPSS-plus score, which takes into account karyotypical abnormalities, platelet count, and transfusion status, reclassified patients with intermediate- $1 / 2$ risk by the DIPSS scores to intermediate-2 or high-risk category. Although the DIPSS-plus has a superior prognostic indication, the expected overall survival predicted by this model still understimates the dismal prognosis of patients with 3q26.2 rearrangement.

Of note, to compare the impact of 3q26.2 rearrangement vs other karyotypical abnormalities in disease progression and patient outcome, we identified a control group of a total of 180 patients with classical Philadelphia chromosome-negative myeloproliferative neoplasms, including 25 patients with essential thrombocythemia, 127 with primary myelofibrosis and 28 with polycythemia vera. These patients developed karyotypical abnormalities other than 3q26.2 rearrangement and the exact time of emergence of the karyotypical abnormalities was available for us. A total of 45/180 (25\%) patients in the control group developed blast crisis at emergence of the karyotypical abnormalities or later compared with 12/15 (80\%) patients with 3q26.2 rearrangement $(P<0.0001)$. The median survival time of patients in the control group from the emergence of the karyotypical abnormalities was 35 months vs 3 months in those with 3q26.2 rearrangement $(P<0.0001)$.

In summary, 3q26.2 rearrangement is a fatal event in the clinical course of patients with classical Philadelphia chromosome-negative myeloproliferative neoplasms that is often associated with a rapid disease progression, blastic transformation, and a dismal outcome. These patients have no response to any current chemotherapy regimens, and new therapeutic options including targeted therapy are needed.

\section{Disclosure/conflict of interest}

The authors declare no conflict of interest.

\section{References}

1 Barbui T, Barosi G, Birgegard G, et al. Philadelphianegative classical myeloproliferative neoplasms: critical concepts and management recommendations from European LeukemiaNet. J Clin Oncol 2011;29:761-770.

2 Saeidi K. Myeloproliferative neoplasms: current molecular biology and genetics. Crit Rev Oncol Hematol 2016;98:375-389.

3 Thiele J, Kvasnicka HM, Orazi A et al. Myeloproliferative neoplasms. In: Swerdlow SCE, Harris NL, Jaffe ES, et al. (eds). WHO Classification of Tumours of Haematopoietic and Lymphoid Tissues. IARC Press: Lyon, France, 2008, pp 31-48.

4 Tefferi A, Pardanani A. Myeloproliferative neoplasms: a contemporary review. JAMA Oncol 2015;1:97-105.

5 Bose P, Verstovsek S. The evolution and clinical relevance of prognostic classification systems in myelofibrosis. Cancer 2016;122:681-692.

6 Ortmann CA, Kent DG, Nangalia J, et al. Effect of mutation order on myeloproliferative neoplasms. N Engl J Med 2015;372:601-612.

7 Cui W, Sun J, Cotta CV, et al. Myelodysplastic syndrome with inv(3)(q21q26.2) or t(3;3)(q21;q26.2) has a high risk for progression to acute myeloid leukemia. Am J Clin Pathol 2011;136:282-288.

8 Lundberg P, Karow A, Nienhold R, et al. Clonal evolution and clinical correlates of somatic mutations in myeloproliferative neoplasms. Blood 2014;123: 2220-2228.

9 Rogers HJ, Vardiman JW, Anastasi J, et al. Complex or monosomal karyotype and not blast percentage is associated with poor survival in acute myeloid leukemia and myelodysplastic syndrome patients with inv(3) (q21q26.2)/t(3;3)(q21;q26.2): a Bone Marrow Pathology Group study. Haematologica 2014;99:821-829.

10 Sun J, Konoplev SN, Wang X, et al. De novo acute myeloid leukemia with inv(3)(q21q26.2) or $\mathrm{t}(3 ; 3)$ (q21;q26.2): a clinicopathologic and cytogenetic study 
of an entity recently added to the WHO classification. Mod Pathol 2011;24:384-389.

11 Wang W, Cortes JE, Lin P, et al. Clinical and prognostic significance of $3 \mathrm{q} 26.2$ and other chromosome 3 abnormalities in CML in the era of tyrosine kinase inhibitors. Blood 2015;126:1699-1706.

12 Wang W, Cortes JE, Tang G, et al. Risk stratification of chromosomal abnormalities in chronic myelogenous leukemia in the era of tyrosine kinase inhibitor therapy. Blood 2016;127:2742-2750.

13 Hussein K, Van Dyke DL, Tefferi A. Conventional cytogenetics in myelofibrosis: literature review and discussion. Eur J Haematol 2009;82:329-338.

14 De Braekeleer M, Le Bris MJ, De Braekeleer E, et al. 3q26.2/EVI1 rearrangements in myeloid hemopathies: a cytogenetic review. Future Oncol 2015;11:1675-1686.

15 Nucifora G. The EVI1 gene in myeloid leukemia. Leukemia 1997;11:2022-2031.

16 Haltrich I, Kost-Alimova M, Kovacs G, et al. Identification of 3q21q26.2 syndrome by "multipoint" interphase FISH analyses in childhood myeloid leukemia. Magy Onkol 2005;49:141-147.

17 Shaffer L, McGowan-Jordan J, Schmid M. An international system for human cytogenetic nomenclature (ISCN 2013), recommendations of the international standing commitee on human cytogenetic nomenclature. Karger: Unionville, USA, 2016.

18 Gangat N, Caramazza D, Vaidya R, et al. DIPSS plus: a refined Dynamic International Prognostic Scoring System for primary myelofibrosis that incorporates prognostic information from karyotype, platelet count, and transfusion status. J Clin Oncol 2011;29:392-397.

19 Yuasa H, Oike Y, Iwama A, et al. Oncogenic transcription factor Evi1 regulates hematopoietic stem cell proliferation through GATA-2 expression. EMBO J 2005;24:1976-1987.

20 Soderholm J, Kobayashi H, Mathieu C, Rowley JD, Nucifora G. The leukemia-associated gene MDS1/EVI1 is a new type of GATA-binding transactivator. Leukemia 1997;11:352-358.

21 Peeters P, Wlodarska I, Baens M, et al. Fusion of ETV6 to MDS1/EVI1 as a result of $t(3 ; 12)(q 26.2 ; p 13)$ in myeloproliferative disorders. Cancer Res 1997;57: 564-569.

22 Groschel S, Sanders MA, Hoogenboezem R, et al. A single oncogenic enhancer rearrangement causes concomitant EVI1 and GATA2 deregulation in leukemia. Cell 2014;157:369-381.

23 Stein S, Ott MG, Schultze-Strasser S, et al. Genomic instability and myelodysplasia with monosomy 7 consequent to EVI1 activation after gene therapy for chronic granulomatous disease. Nat Med 2010;16: 198-204.

24 Fonatsch C, Gudat H, Lengfelder E, et al. Correlation of cytogenetic findings with clinical features in 18 patients with inv(3)(q21q26.2) or $\mathrm{t}(3 ; 3)(\mathrm{q} 21 ; \mathrm{q} 26.2)$. Leukemia 1994;8:1318-1326.

25 Kennedy JA, Atenafu EG, Messner HA, et al. Treatment outcomes following leukemic transformation in Philadelphia-negative myeloproliferative neoplasms. Blood 2013;121:2725-2733.

26 Mesa RA, Li CY, Ketterling RP, et al. Leukemic transformation in myelofibrosis with myeloid metaplasia: a single-institution experience with 91 cases. Blood 2005;105:973-977.

27 Groschel S, Sanders MA, Hoogenboezem R, et al. Mutational spectrum of myeloid malignancies with inv(3)/t(3;3) reveals a predominant involvement of RAS/RTK signaling pathways. Blood 2015;125: 133-139.

28 Lavallee VP, Gendron P, Lemieux S, et al. EVI1-rearranged acute myeloid leukemias are characterized by distinct molecular alterations. Blood 2015;125:140-143.

29 Tam CS, Abruzzo LV, Lin KI, et al. The role of cytogenetic abnormalities as a prognostic marker in primary myelofibrosis: applicability at the time of diagnosis and later during disease course. Blood 2009;113:4171-4178.

30 Passamonti F, Cervantes F, Vannucchi AM, et al. A dynamic prognostic model to predict survival in primary myelofibrosis: a study by the IWG-MRT (International Working Group for Myeloproliferative Neoplasms Research and Treatment). Blood 2010;115: 1703-1708. 